\title{
Impact of the Ebola epidemic on general and HIV care in Macenta, Forest Guinea, 2014
}

\author{
David Leuenberger $^{\mathrm{a}}$, Jean Hebelamou ${ }^{\mathrm{a}}$, Stefan Strahm ${ }^{\mathrm{a}}$, \\ Nathalie De Rekeneire $^{b, c}$, Eric Balestre ${ }^{b, c}$, Gilles Wandeler ${ }^{d, e, f}$, \\ François Dabis ${ }^{\mathrm{b}, \mathrm{c}}$, for the leDEA West Africa study group
}

\begin{abstract}
Objective: The current Ebola epidemic massively affected the Macenta district in Forest Guinea. We aimed at investigating its impact on general and HIV care at the only HIV care facility in the district.

Design: Prospective observational single-facility study.

Methods: Routinely collected data on use of general hospital services and HIV care were linked to Ebola surveillance data published by the Guinea Ministry of Health. In addition, we compared retention among HIV-infected patients enrolled into care in the first semesters of 2013 and 2014.

Results: Throughout 2014, service offer was continuous and unaltered at the facility. During the main epidemic period (August-December 2014), compared with the same period of 2013, there were important reductions in attendance at the primary care outpatient clinic $(-40 \%)$, in HIV tests done $(-46 \%)$, in new diagnoses of tuberculosis $(-53 \%)$ and in patients enrolled into HIV care $(-47 \%)$. There was a smaller reduction in attendance at the HIV follow-up clinic $(-11 \%)$. Kaplan-Meier estimates of retention were similar among the patients enrolled into care in 2014 and 2013. In a multivariable Cox regression analysis, the year of enrolment was not associated with attrition (hazard ratio 1.02; 95\% confidence interval: $0.72-1.43$ ).

Conclusion: The Ebola epidemic resulted in an important decrease in utilization of the facility despite unaltered service offer. Effects on care of HIV-positive patients enrolled prior to the epidemic were limited. HIV care in such circumstances is challenging, but not impossible.

Copyright (c) 2015 Wolters Kluwer Health, Inc. All rights reserved.
\end{abstract}

AIDS 2015, 29:1883-1887

Keywords: Ebola epidemic, Guinea, HIV care, primary healthcare

\section{Introduction}

Since early 2014, the worst Ebola virus epidemic in human history has affected a number of West African countries [13]. Macenta district (298282 inhabitants [4]) has so far been the worst hit area in Guinea. As of 31 December 2014, 743 cases were reported ([5], cumulative incidence: 249 cases/ 100000 inhabitants). In Macenta, the peak monthly incidence was reached in September 2014, at 79 cases/ 100000 inhabitants (Fig. 1a and b).

\footnotetext{
${ }^{a}$ Centre Médical de Macenta, Mission Philafricaine, Conakry, Guinea, bINSERM U897 - Centre Inserm Epidémiologie et Biostatistique, Université de Bordeaux, 'Université de Bordeaux, ISPED, Centre INSERM U897-Epidemiologie-Biostatistique, Bordeaux, France, dDepartment of Infectious Diseases, University Hospital Bern, e Institute of Social and Preventive Medicine, University of Bern, Bern, Switzerland, and ' Department of Infectious Diseases, University of Dakar, Senegal.

Correspondence to Dr David Leuenberger, Mission Philafricaine, B.P. 214, Conakry 1, Rép. de Guinée.

E-mail: david.leuenberger@sam-ame.org

Received: 30 April 2015; revised: 9 June 2015; accepted: 16 June 2015.
} 
(a)

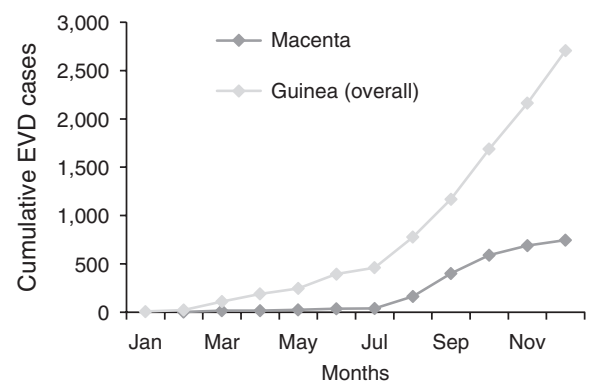

(b)

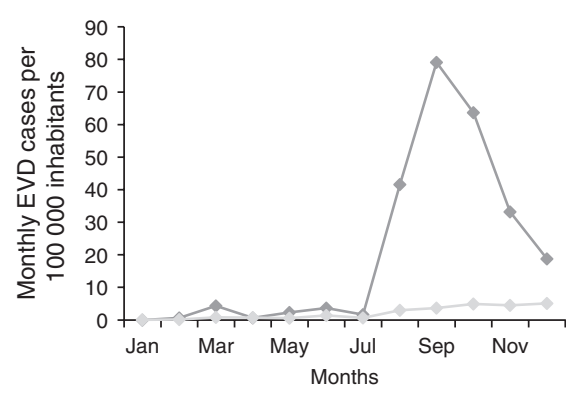

Fig. 1. Confirmed and probable Ebola virus disease cases reported by the Guinea Ministry of Health, 2014. Cumulative case load (a) and monthly incidence per 100000 inhabitants (b) for the whole country and for the district of Macenta. EVD, Ebola virus disease.

In addition to the morbidity and mortality owing to infection with the Ebola virus itself, the current epidemic has brought a number of additional burdens on the affected population [6,7]. Several anecdotal reports have speculated on the likelihood of negative effects of the epidemic on established treatment programs (such as for malaria [8]), leading in turn to a probable rise in nonEbola-related health problems [9]. Previous reports have documented the impact of man-made threats on HIV care $[10,11]$. The present epidemic is an opportunity to look at the impact of a major threat of natural origin on HIV care [12]. Early reports from urban settings in Monrovia, Liberia and Conakry, Guinea [13,14] have suggested a negative impact on clinic attendance and retention. We aimed at investigating the impact of the Ebola epidemic on general and HIV services at the Centre Médical, a public-private partnership between the Guinean Ministry of Health and Public Hygiene $(\mathrm{MoH})$ and a Switzerland-based nongovernmental organization, located in Macenta and serving a rural population.

\section{Methods}

The Centre Médical is a specialized hospital established in 1981. It serves as a regional referral center for leprosy, tuberculosis (TB) and HIV/AIDS. In addition, it offers primary healthcare services to the local population through a general outpatient department (OPD). Overall, there were 13133 outpatient visits in 2014. The HIV/ AIDS department is currently the only functioning HIV care facility in the whole district. Most patients enter the system through the OPD. All services directly related to HIV/AIDS care are free of charge. The hospital is funded by the $\mathrm{MoH}$, private donors and through revenues generated by service fees and drug sales to the general population.

Several sets of hospital data were used: data collected routinely and prospectively for hospital planning purposes as well as for reports required by health authorities; internal accountancy data, that is the hospital revenues from service fees and drug sales; data collected as part of the International epidemiological Databases to Evaluate AIDS (IeDEA) West Africa collaboration, a large network of HIV cohorts in West Africa, described previously [15]. Briefly, this collaboration aims at evaluating the effectiveness of long-term antiretroviral therapy (ART) in West Africa and at documenting the morbidity burden in HIVinfected individuals. This study was approved by the Guinean National Committee for Health Research Ethics. All participants provided a written informed consent. For the purpose of this analysis, we linked these hospital data to Ebola surveillance data published daily by the $\mathrm{MoH}$ together with the World Health Organization (WHO).

We chose the monthly number of OPD visits and HIV tests as well as diagnoses of TB and HIV infections as measures of use of general hospital services. Impact on hospital management was assessed through the monthly hospital revenue from service fees and drug sales. Impact on the HIV program was assessed through the monthly number of HIV tests done, HIV infections diagnosed, HIV-positive patients enrolled into care, and visits at the HIV follow-up clinic. We compared these indicators between 2013 and 2014.

In addition, we compared retention in care between HIV patients enrolled into care during the first semesters of 2013 (outside of the epidemic) and 2014 (before the main epidemic period), using Kaplan-Meier estimates (with censoring for patients transferred to other facilities). To increase the sensitivity of our analysis for short-term attrition, we used a stringent 30-day lateness after next scheduled visit-definition for loss to follow-up (LTFU). We analyzed factors associated with attrition (defined as either death or LTFU) using univariable and multivariable Cox regression models (adjusted for sex, age, geographical origin, mode of entry, initial WHO stage, initial $\mathrm{CD} 4^{+}$count, presence of TB at enrolment, cotrimoxazole prophylaxis and ART status). Statistical analyses were done with SAS version 9.2 (SAS Intitiute, Cary, North Carolina, USA). 


\section{Results}

There was one Ebola-related death among the personnel (head of laboratory). Throughout 2014, all hospital services were fully staffed and functioning at all times.

An important and sustained reduction in general OPD attendance was documented in August-December 2014 (the main epidemic period in Macenta), compared with the same period in 2013 (-40\%; Fig. 2a). OPD attendance improved slowly between September and December 2014, paralleling the downward trend of new Ebola cases.

The drop in OPD attendance resulted in a 53\% reduction in new TB diagnoses (Fig. 2b), a 46\% drop in HIV tests done (Fig. 2c) and a 53\% drop in positive HIV tests

(a)

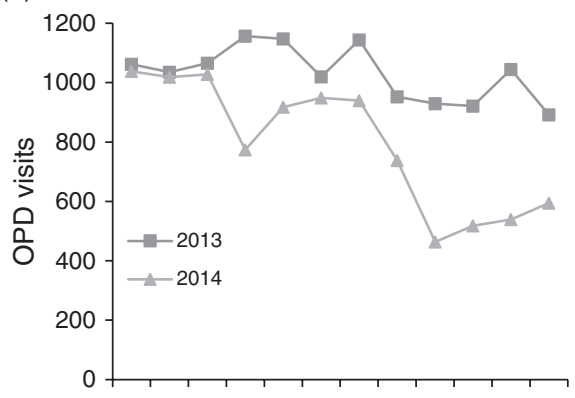

(c)

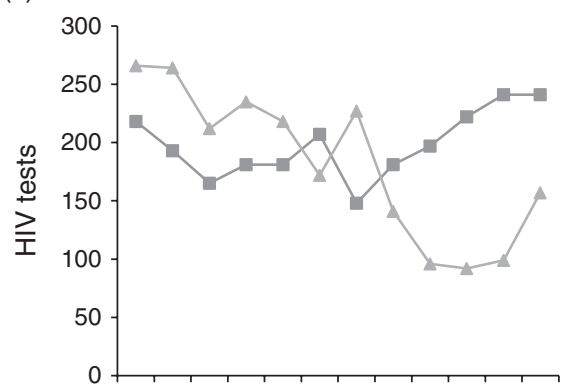

(e)

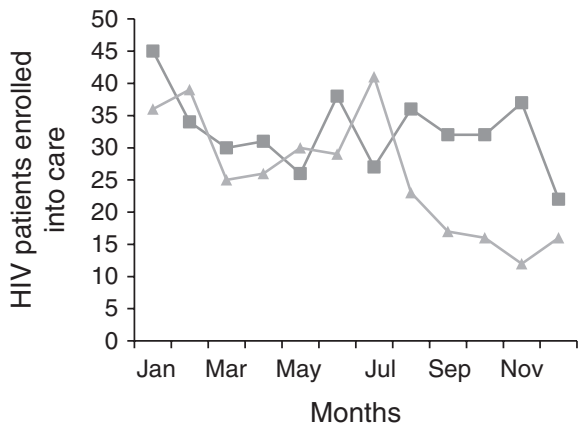

between August and December 2014 compared with the same period of 2013.

The drop in general OPD attendance resulted in a major reduction in self-generated hospital revenues, from 56850 US\$ in August-December 2013 to 32450 US\$ during the same period in 2014 (-43\%, Fig. 2d).

We noted a massive drop in new enrolments of HIVinfected patients into chronic care at the Centre Médical during the epidemic period: $-47 \%$ from August to December 2014 compared with the same period of 2013 (Fig. 2e). However, we only noted a marginal reduction in the number of follow-up visits at the HIV clinic $(-11 \%)$, with no clear month-by-month pattern (Fig. 2f). We documented three deaths because of Ebola virus disease among the patients followed. Regarding

(b)

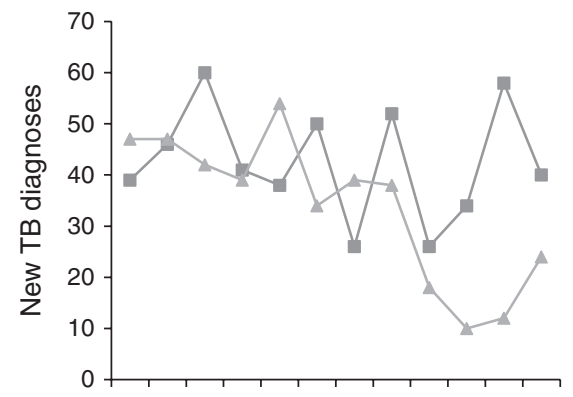

(d)

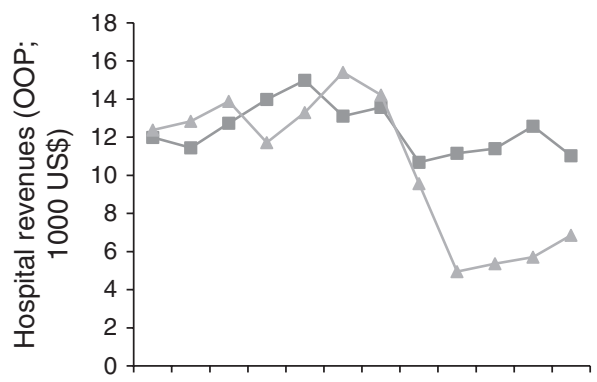

(f)

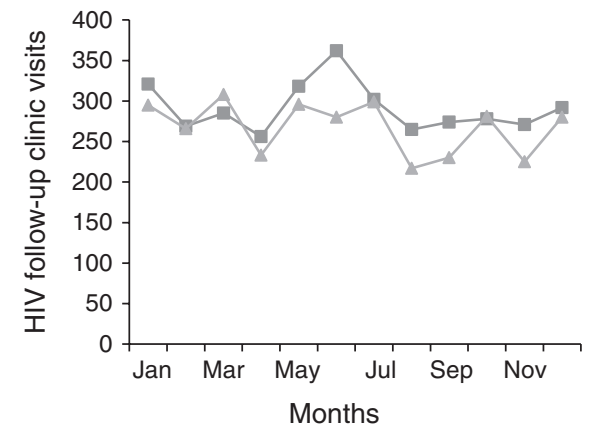

Fig. 2. Selected monthly indicators of general and HIV care at the Centre Médical, Macenta, 2013-2014. (a) Primary care outpatient department (OPD) attendance. (b) Patients newly diagnosed with tuberculosis (TB). (c) HIV tests done. (d) Self-generated hospital revenues (out-of-pocket patient expenditures for service fees and drug purchases). (e) Patients enrolled into HIV care. (F) HIV follow-up clinic attendance. OOP, out-of-pocket patient expenditures; OPD, outpatient department; TB, tuberculosis. 
ART dispensation, there was an increase of 26\% in 2014 compared with 2013 (4864 patient-months dispensed in 2013 for 675 patients; 6143 in 2014 for 780 patients).

Compared with patients enrolled in the first semester 2013, those enrolled in the first semester 2014 were less likely to be diagnosed on site, to have TB and to be on cotrimoxazole prophylaxis than those enrolled a year earlier. Overall, ART use was comparable, although a history of ART prior to arrival at the Centre Médical was more common in 2014 (see table, Supplemental Digital Content 1, http://links.lww.com/QAD/A734). Kaplan-Meier estimates of retention in care showed no difference between the two cohorts $(P=0.43$; see figure, Supplemental Digital Content 2, http://links.lww.com/ QAD/A734). In the multivariable Cox regression analysis, the following factors were independently and positively associated with attrition: presence of active TB at enrolment, initial WHO status 3 or 4 , initial CD4 counts below 350 cells/ $\mu$ l, and not starting ART (see table, Supplemental Digital Content 3, http://links.lww. com/QAD/A734). Being enrolled into care in 2014 was not associated with a higher risk of attrition (hazard ratio $1.02,95 \%$ confidence interval $0.72-1.43$ ).

\section{Discussion}

We studied the impact of the Ebola epidemic on the use of general hospital services in one of the worst hit districts of West Africa and documented its negative impact on attendance at a primary care clinic. A steep drop in new $\mathrm{TB}$ and HIV/AIDS diagnoses was shown as well as a reduction in enrolment for HIV care, which were directly correlated to Ebola occurrence in the district. As the service fees and drug purchases by OPD patients are an important part of hospital revenues, a marked direct financial impact on general hospital management was also noted. However, retention of HIV-infected patients already in active follow-up when the epidemic started was only marginally affected.

The effects of the current Ebola epidemic went far beyond Ebola-related morbidity and mortality. The decreased utilization of our healthcare facility was not an isolated pattern $[16,17]$. Two commonly cited reasons - reduction in service offer [13,17] or fear from contracting Ebola virus itself - did not apply in our setting, as service offer was continuous and unaltered, and the Centre Médical never served as an Ebola treatment unit. As there was no detectable shift of patient attendance toward other healthcare facilities in our district, we hypothesize that many patients were either forgoing treatment for their ailments altogether, or using private services such as private pharmacies, with the concurrent well known problems of high fees, unregulated care, poor-quality medical advice and substandard drugs. This might have led to an increase in Ebola-unrelated morbidity and mortality in the Macenta district. Unstructured interviews with local authorities and patients attending our hospital highlighted the generalized fear in the population of being isolated as a suspected Ebola case when attending a hospital. In addition, even before the Ebola epidemic, we had observed a trend toward decreased levels of trust by the general population in the generally weak healthcare system in Guinea.

The analysis of the financial consequences of the Ebola epidemic on our institution highlighted another important problem affecting a healthcare system largely dependent on out-of-pocket-expenditures from patients. In such a situation, a drop in attendance (irrespective of the reason) leads to potentially serious financial difficulties for a healthcare facility, which undermines the continuous service offer. In our context, continuous delivery of health services was only possible because the Centre Médical was not entirely dependent on such revenues, and because some private international donors accepted to finance an extraordinary budget assistance directly to the Centre Médical.

Despite the dramatic effects of the Ebola epidemic on general medical care in our setting, we did not observe any change in retention in care of HIV-infected patients in active follow-up throughout the peak of the epidemic. This was unexpected for several reasons: the same fears described above could apply to HIV-infected patients followed at the Centre Médical; a substantial proportion of HIV-infected patients followed at the Centre Médical lives outside Macenta district - at a time when Macenta was much more affected by the Ebola epidemic than their district of origin, they could have sought treatment elsewhere; the Ebola epidemic led to a general economic downturn in Guinea [6], which could have stopped patients from travelling from the villages to Macenta town for their clinic visits. Although we cannot be sure of the main reason(s) that led retention in care to remain fairly stable throughout the epidemic, trust might have played a major role at our institution. The staff of the Centre Médical seems to have managed to foster enough trust in enrolled patients to allow them to overcome the fears prevalent in the general population.

The main strength of our study is the good quality of primary data routinely collected identically before and during the epidemic. The main limitations are the absence of data on both the reasons and the consequences of the observed reductions in health system use.

Overall, our study highlights the dramatic impact of the Ebola epidemic on hospital attendance in Guinea, with the likely public health consequences. Conversely, it suggests that long-term HIV care, though challenging, is not altogether impossible, even in the setting of an uncontrolled Ebola epidemic, as long as there is no reduction in hospital service offer. 


\section{Acknowledgements}

We thank all the staff of the Centre Médical, especially Dr Eric Bafende (hospital director up to August 2014) and his successor Dr Ismaël Béavogui for their support.

\section{Contributions}

This study was sponsored by the National Cancer Institute (NCI), the Eunice Kennedy Shriver National Institute of Child Health \& Human Development (NICHD) and the National Institute of Allergy and Infectious Diseases (NIAID) of the U.S. National Institutes of Health (NIH), as part of the International Epidemiologic Databases to Evaluate AIDS (IeDEA) grant (Award Number U01AI069919). The content is solely the responsibility of the authors and does not necessarily represent the official views of the National Institutes of Health.

The sponsor of the study had no role in study design, data collection, data analysis, data interpretation, or writing of the report. All the authors had full access to all the data in the study. D.L. and F.D. had final responsibility for submitting the article for publication.

D.L., J.H. and S.S. live in Macenta. They were in charge of the general and HIV care at the Centre Médical throughout 2014 and collected the data. D.L., G.W. and F.D. designed the study. D.L. wrote the first draft. E.B. performed the statistical analysis. All the authors contributed to the revisions of the manuscript, and have approved the final version.

\section{Conflicts of interest}

D.L. declares receiving a travel grant from Gilead Sciences Switzerland to present parts of the results of this study at the 2015 Conference on Retroviruses and Opportunistic Infections. The other authors declare no conflicts of interest.

\section{References}

1. Bausch DG, Schwarz L. Outbreak of Ebola Virus disease in Guinea: where ecology meets economy. PLoS Negl Trop Dis 2014; 8:e3056.
2. Piot P, Muyembe JJ, Edmunds WJ. Ebola in West Africa: from disease outbreak to humanitarian crisis. Lancet 2014; 14:10341035.

3. Baize S, Pannetier D, Oestereich L, Riger T, Koivogui L, Magassouba $\mathrm{N}$, et al. Emergence of Zaire Ebola virus disease in Guinea - preliminary report. N Engl / Med 2014; 371:14181425.

4. Institut National de la Statistique. Résultats préliminaires du troisième recensement général de la population et de l'habitation. Conakry, Guinea. 2014. http://www.stat-guinee.org/ index.php/result-prelim-rgph3. [Accessed 20 March 2015].

5. Guinea Ministry of Health /WHO. Daily situation report 31/12/ 2014.

6. World Bank. Update on the economic impact of the 2014 Ebola epidemic on Liberia, Sierra Leone, and Guinea. Washington, DC: World Bank Group. 2014. http://documents.worldbank. org/curated/en/2014/12/20454884/update-economic-impact2014-ebola-epidemic-liberia-sierra-leone-guinea. [Accessed 20 March 2015].

7. UN News Centre. Ebola: UN special envoy says combating stigma integral to overall crisis response (12/11/2014). http:// www.un.org/apps/news/story.asp? NewsID $=49320 \# . V Q 0 s V r$ Vyb4 g. [Accessed 20 March 2015].

8. Hayden EC. Ebola obstructs malaria control. Nature 2014; 514:15-16.

9. Kieny MP, Evans DB, Schmets G, Kadandale S. Health-system resilience: reflections on the Ebola crisis in western Africa. Bull World Health Org 2014; 92:850.

10. Vreeman RC, Nyandiko WM, Sang E, Musick BS, Braitstein P, Wiehe SE. Impact of the Kenya postelection crisis on clinic attendance and medication adherence for HIV-infected children in western Kenya. Confl Health 2009; 3:5doi: 10.1186/ 1752-1505-3-5.

11. Yoder RB, Nyandiko WM, Vreeman RC, Ayaya SO, Gisore PO, Braiststein $\mathrm{P}$, et al. Long-term impact of the Kenya postelection crisis on clinic attendance and medication adherence for HIVinfected children in western Kenya. I Acquir Immune Defic Syndr 2012; 59:199-206.

12. Wainberg MA, Kippax S, Bras M, Sow PS. HIV and Ebola: similarities and differences. J Int AIDS SoC 2014; 17:19896.

13. Tattevin P, Baysah MK, Raguin G, Toomey J, Chapplain JM, Taylor ME, et al. Retention in care for HIV-infected patients in the eye of the Ebola storm: lessons from Monrovia, Liberia. AIDS 2015; 29:N000-N10.

14. Ndawinz JDA, Cissé M, Diallo MSK, Sidibé CT, Dortenzio E. Prevention of HIV spread during the Ebola outbreak in Guinea. Lancet 2014; 385:1393.

15. Egger M, Ekouevi DK, Williams C, Lyamuya RE, Mukumbi $\mathrm{H}$, Braitstein $\mathrm{P}$, et al. Cohort profile: The international epidemiological databases to evaluate AIDS (IeDEA) in sub-Saharan Africa. Int / Epidemiol 2012; 41:1256-1264doi:10.1093/ije/ dyr080.

16. Delamou A, Hammonds RM, Caluwaerts S, Utz B, Delvaux T. Ebola in Africa: beyond epidemics, reproductive health in crisis. Lancet 2014; 384:2105.

17. Médecins Sans Frontières. Sierra Leone: MSF suspends emergency pediatric and maternal services in Gondama (16/10/2014). http://www.doctorswithoutborders.org/article/sierra-leone-msfsuspends-emergency-pediatric-and-maternal-services-gondama. [Accessed 20 March 2015). 